\title{
THE MANAGEMENT OF PATIENTS WITH CHRONIC GASTRIC ULCER
}

\author{
By RODNEY MAINGOT, F.R.C.S.(Eng.), Hon.F.I.C.S.
}

\author{
(Senior Surgeon to the Southend General Hospital, and to the Royal Waterloo Hospital, London; \\ Consultant Surgeon, Essex County Council; Group Adviser, E.M.S.)
}

All too frequently in medical literature we find chronic gastric and chronic duodenal ulcer classified together as being one disease-peptic ulcer. This, in my opinion, is a very grave mistake, for although it is readily admitted that they have certain aetiological features in common, the risk of malignant transformation, the degree of morbidity, the potentialities of disaster, and the accompanying disturbances are factors which differ widely according to whether the case is one of duodenal ulcer or of chronic ulcer of the stomach. Again, the medical and especially the surgical methods of treatment of the two conditions vary in many essential details.

A chronic gastric ulcer may become malignant, whilst cancerous degeneration of a duodenal ulcer is a pathological curiosity. The incidence of malignant change in gastric ulcers has been computed to be between Io and 20 per cent. Professor Stewart of Leeds ${ }^{12}$ who has made an extensive study of ulcer-cancer, concludes that in 9.5 per cent of cases a chronic ulcer becomes carcinomatous, and that carcinoma originates in a chronic ulcer in I7 per cent. Katschio reported an incidence of 20 per cent; Finsterer 8 in his Address before the Royal Society of Medicine stated that in 532 cases of resection for gastric ulcer the ulcer was carcinomatous in I4I instances-20 per cent. Chamberlain 3 in a follow-up study of $2 \mathrm{I} 6$ patients treated for suspected simple gastric ulcer found that 9.5 per cent of this group had died of cancer of the stomach. Waltman Walters and Cleveland15 found that in Ioo consecutive cases of reported "gastric ulcer" upon which they operated the lesion was proved to be cancerous on microscopical investigation in I9. In my last 50 cases of sub-total gastrectomy for supposedly simple ulcer of the stomach no fewer than II of these were shown on microscopical investigation to be undergoing malignant change.

At the present time it is generally agreed that it is impossible by clinical methods, by gastroscopic or by barium meal X-ray examination to distinguish positively simple gastric ulcer from early carcinomatous ulcer. Eusterman and Balfour7 in their monograph, "The Stomach and Duodenum," write:-

"There is as yet no single sign or any combination of signs which will determine with absolute certainty whether or not a gastric ulcer is malignant."

If the above statements are correct, namely, that some ro-20 per cent of gastric ulcers undergo malignant transformation and that a chronic gastric ulcer cannot be distinguished from an early ulcerating carcinoma of the stomach by any means at our disposal apart from microscopical enquiry or the therapeutic test, which we shall later discuss, then it must be conceded:-

(I) That as gastric ulcer is a very treacherous disease, all patients suffering from this lesion should be promptly admitted to hospital for investigation and treatment:

(2) That so-called ambulatory treatment for ulcer cases is dilatory, unscientific and dangerous, and cannot in any circumstances be condoned;

(3) That if medical treatment fails to bring about a cure of the ulcer after a fair trial, operative measures should be invoked;

(4) That although medical therapy is highly efficacious for the simple uncomplicated lesion, operative interference will nevertheless be called for more frequently in the case of chronic gastric ulcer than of chronic duodenal ulcer. This fact is confirmed by the figures of the Mayo Clinic, which show that in 1939 there were 207 patients in whom a diagnosis of gastric ulcer was made, of which rog were operated upon, an incidence of $52 \cdot 6$ per cent. In the same year a diagnosis of duodenal ulcer was made in 2,729 cases and operation carried out in $467-\mathrm{I} 7 \cdot$ I per cent. Lahey ${ }^{\mathrm{II}}$ writes on this subject as follows:-

"Up to the present time we have treated in this clinic, under bed management, 3,534 patients with ulcer-249 with gastric and 3,285 with duodenal ulcers; included in this number have been II5 patients with gastrojejunal ulcers. In order that our attitude toward the surgical treatment 
of peptic ulcer may be clear, we wish to state that of this entire series only 8 per cent of the patients with duodenal ulcer and but 23 per cent of those with gastric ulcer were submitted to surgery."

\section{SCHEME OF MANAGEMENT}

The patient is admitted to hospital and confined to bed. Rest in bed is an essential part of the treatment. One cannot treat these patients satisfactorily unless they are under constant observation, nor can therapy be adequately applied in their own homes or in nursing institutions which are not suitably equipped.

The history of the case is taken and a routine physical examination is conducted, after which the following investigations are carried out:-(I) Fractional test meal examinations; (2) Tests for occult blood in the stools; (3) Complete examination of the blood; (4) Gastroscopy; (5) Barium meal X-ray examination.

The size, shape, position and other characteristic features of the ulcer are noted and drawn on a special chart. All accessible foci of infection receive attention. If the patient is anaemic, blood transfusions are given, and if he is dehydrated, plasma and also glucose and salt solution are run into a vein by the slow-drip method.

An Einhorn or Ryle tube is passed through the nostril into the stomach, and when its distal bulbous tip is shown to be lying in the duodenum the proximal end is attached by means of a glass tube and rubber connection to a large reservoir containing milk or milk substitute. This container is slung to an upright support about three feet or so above the patient's head. The milk, which is neither warmed nor citrated, is run in at an even slow drip both by day and by night so that about four pints are introduced into the intestine in each 24 hours. The milk drip is continued for three weeks before the tube is withdrawn.

Some patients prefer to have the tube introduced through the mouth rather than through the nose. A portion of the tube is strapped to the side of the face with adhesive tape. The great majority of patients tolerate the tube extremely well, and suffer little or no inconvenience from its presence; but a few of them will not co-operate and these are best treated on the lines laid down by Hurst, Eusterman, Lahey or Sippy.

The patient, with the tube in situ, is encouraged to drink water, barley water, tea, sweetened lime and lemon juice, etc., and is given a mixture of cod-liver oil and belladonna thrice daily, whilst, if the acid values of the gastric juice are found to be unduly high, alkaline draughts are administered 8-hourly. The essential vitamins are also prescribed in adequate doses. The patient may not gain weight on this treatment, but few lose appreciably.

At the end of three weeks the duodenal tube is withdrawn. A barium meal X-ray examination and a gastroscopic investigation are then carried out to see if the ulcer has diminished in size, and the faeces are tested to ascertain whether occult blood is disappearing from the stools. Visual signs of sound and progressive healing call for further perseverance with these methods.

Spriggs ${ }^{14}$ considers that in the average case it takes about three months for a chronic gastric ulcer to heal completely under strict medical supervision. The disappearance of the crater on skiagrams is of course a most helpful and encouraging sign, but it does not necessarily mean that the ulcer has healed. We know too well that carcinomatous processes may extend from the margin of an ulcer into the crater and thus obliterate it, and that small ulcerating cancers, owing to the absorption of inflammatory products (which invariably occurs after a course of stringent medical therapy), may mask their malignant characteristics by shrinking appreciably. The disappearance of the crater as evidenced by X-ray pictures should merely be regarded as a sign that after a course of efficient medical measures the lesion is healing well, that the surrounding oedema is subsiding or has subsided, and that the crater is flattening out by exuberant granulation tissue which will eventually act as a support for the new although inferior mucous membrane which has started to creep slowly across the gap in the gastric.wall.

No patient is regarded as cured until symptoms have ceased altogether, the stools have been free from occult blood for several weeks, the haemoglobin percentage is normal, and radiological and gastroscopic evidences of sound healing are irrefutable.

For the case which shows a gratifying response to this type of therapy, the milk drip treatment is continued for a further three weeks. By the end of this time healing should be well advanced and the indwelling tube can be finally removed. 
At this stage all the investigations and tests already referred to are repeated and if the findings are satisfactory the patient is then treated as an ambulatory ward case and is ordered Hurst's9 post-ulcer regime for six weeks.

After the completion of the three months' course of treatment, final gastroscopic examination is performed to ascertain if the new gastric mucosa which now covers the dangerous area is healthy and staunch. If all is well, the patient is discharged from hospital and is instructed to follow the rules laid down in Hurst's post-ulcer regime as strictly and conscientiously as possible for a further three months.

The scheme of medical management here outlined, which is adapted from Einhorn's methods 6 , therefore entails frequent investigations by means of X-rays and the gastroscope, rest in bed for six weeks with the milk drip working almost continuously, and supervision under strict medical treatment for a further six weeks. This also involves strength of character, a cheerful optimism, whole-hearted co-operation, an unflinching perseverance on the part of the patient, careful and watchful nursing, and minute attention to many details. It can, in my opinion, only be conducted in a thorough manner in a well-equipped hospital which is staffed with a personnel who have made a special study of gastric disorders.

The results are encouraging, as approximately 50 per cent of the patients thus treated can be discharged as cured, and provided they continue to be reasonably careful with regard to diet, mode of living, etc., a large majority of them are eventually restored to full economic efficiency.

\section{INDICATIONS FOR SURGICAL TREATMENT}

(I) After a course of the treatment outlined above, occult blood is still present in the stools and the ulcer niche has not diminished in size.

(2) At the end of six weeks' treatment the ulcer, as observed on gastroscopy and on fluoroscopy, although smaller, has assumed an indolent appearance and traces of blood can still be detected in the faeces.

(3) Following a course of treatment the ulcer has healed but later on during the probationary period there is recurrence of incapacitating symptoms and unmistakable signs of reactivation.

(4) Hour-glass deformity is present and this is associated with malnutrition.

(5) The patient is over the age of 60 , gives a short history of indigestion, and a crater can be visualised in the stomach on X-ray investigation.

(6) In those cases where haemorrhage from the ulcer bed is continuous or intermittent, and is difficult to control, and the blood-loss threatens life.

(7) Ulcers situated on the greater curvature or in the pyloric region, since malignancy cannot here be ruled out.

(8) Multiple chronic gastric ulcers or combined chronic gastric and duodenal ulcer.

(9) Chronic gastric ulcer associated with chronic duodenal ileus.

(I0) Very large ulcers - with a diameter of one inch or more-which have penetrated deeply into the substance of the pancreas or liver, as here medical treatment is not likely to prove availing and the presence of cancer in the margin of the crater is always difficult to refute.

(II) Expedient circumstances and economic reasons in exceptional cases.

\section{OPERATIONS FOR GASTRIC ULCER}

The following is a list of some of the operations which are nowadays performed for chronic gastric ulcer:-

(I) Excision of the ulcer alone.

(2) Gastro-jejunostomy alone.

(3) Excision of the ulcer combined with gastro-jejunostomy.

(4) Sleeve resection.

(5) Partial gastric exclusion.

(6) Partial gastrectomy: (a) Billroth I types of repair; (b) Polya methods with or without the Hofmeister valve. 


\section{OBSERVATIONS ON THE OPERATIONS FOR GASTRIC ULCER}

No useful purpose would be served by describing a number of obsolete procedures, such as cholecysto-gastrostomy, the Moynihan I operation, the Roux-in-Y method, transgastric excision of a posterior-wall ulcer, and the like. The great number of names and techniques associated with gastric surgery is a source of considerable confusion. The fact that a certain operation has been performed at some surgical clinic or by a famous surgeon does not necessarily make it valuable. It is agreed by all authorities that the ideal operation for chronic gastric ulcer is partial gastrectomy, but when owing to the poor general condition of the patient the risks of a radial resection appear to be prohibitive, the surgeon yet has the choice of other procedures, from which, however, he will need to select with wise discrimination.

The objects of any operation for chronic gastric ulcer may be briefly stated as follows:(I) Wide removal of the lesion; (2) Permanent alteration of the motor and secretory functions of the stomach; (3) Assurance of relief of symptoms; and (4) Protection against recurrence of ulceration and against the subsequent development of cancer of the stomach.

The success or failure of operative treatment for gastric ulcer depends to a great extent upon:-(I) The judicious selection of the patient for operation; (2) Careful pre-operative treatment; (3) A wise choice of surgical procedure for the individual case; (4) The experience of the surgeon and the technique employed; (5) A skilfully administered anaesthetic; (6) The method adopted in the conduct of the after-care; and (7) Unexpected complications.

There is as yet no routine surgical procedure for chronic gastric ulcer. An operation which may be considered advisable in one case may be unnecessarily dangerous or totally inadequate in another. The best results follow those measures which are directed to a wide excision of the lesion, the overcoming of stasis and the reduction of gastric acidity. This is brought about by a variety of operations, but by far the best and most widely practised is partial gastrectomy.

The standards of surgical treatment should be based upon the course which offers the optimum results in competent hands. With increasing experience as regards the indications, contra-indications, the difficulties, the technical methods of overcoming certain dangers, and the factors which make for safety, I find that the operation of choice-partial gastro-duodenal resection-is applicable in about 95 per cent of my own cases.

I shall now discuss the advantages and disadvantages of the operations which are most generally performed for cases of chronic gastric ulcer.

\section{(r) Simple Excision of the Ulcer.}

It would indeed be an easy solution of the ulcer problem if excision of the ulcer alone could be depended upon to cure every case. Unfortunately, this operation is followed by recurrence of ulceration or by severe crippling symptoms in over half the cases. This statement is confirmed by the B.M.A. Report, I930, which was submitted by Dr. Luff. Excision of the ulcer guards the patient against perforation, haemorrhage, mid-gastric constriction and the threat of malignant invasion, and is associated with a low mortality; but it does not reduce gastric acidity, it does not overcome stasis, nor does it relieve pylorospasm, and there is only a 50 per cent chance of even tolerably good results following upon this type of surgical procedure.

Nevertheless, if simple excision can be carried out without interfering with the motor functions of the stomach and without the suture line encroaching upon the greater or lesser curvature or the pyloric segment of the stomach, it then has a sphere of usefulness, e.g., in obese patients, in those who are very debilitated, and in those who are not in a condition to withstand a more extensive undertaking. If all these requirements are fulfilled, this operation will still have a place-if a decidedly limited one-in the management of chronic gastric ulceration.

\section{(2) Gastro-Jejunostomy Alone.}

Gastro-jejunostomy alone is very rarely indicated for the type of lesion under discussion. It may, however, be called for when the patient has a large penetrating ulcer situated high up on the lesser curvature close to the cardia, associated with extensive surrounding inflammation and a severe degree of pylorospasm, and when resection is impossible owing to the local conditions and the poor state of the patient's health.

With these large inaccessible cardial ulcers it would on the whole appear advisable to continue with medical treatment for some weeks rather than immediately to attempt an indirect 
operation, the results of which will be equivocal. The greater the experience of the surgeon, the fewer indirect operations will he perform for chronic gastric ulcer. The experienced surgeon knows full well that ulcers which on first sight appear to be fixed and which are situated high up on the lesser curvature or on the posterior wall close to the cardiac orifice can often be excised after the viscus has been thoroughly mobilised and the lesion separated from its attachments posteriorly. Frequently these cardial lesions appear higher in the skiagrams than is actually the case, this being due to the fore-shortening of the stomach proximally which is caused by perforation of the ulcer into the pancreas or into the gastro-hepatic omentum. In most of these cases ample stomach can be found above the lesion for safe partial gastric resection after mobilisation of the stomach and ligation of the gastro-hepatic omentum. This point receives special emphasis from Waltman Walters. ${ }^{16}$

The safety of gastro-jejunostomy should not lead the surgeon to disregard the added advantage of removal of the lesion whenever this is feasible, for, following gastro-jejunostomy alone for gastric ulcer, there are several factors of greater importance than immediate mortality, particularly in regard to malignant degeneration.

\section{(3) Excision of the Ulcer Combined with Gastro-Jejunostomy.}

This operation yields better results than simple excision alone or gastro-jejunostomy alone; but it is being performed less and less frequently and has been in large measure superseded by the Polya methods.

By this plan the ulcer is excised by wedge- or V-excision, using a knife, scissors or cautery, or if the lesion is small it may be destroyed by heat on the lines originally suggested by Balfour $(\mathrm{I}$ and 2$)$, after which posterior gastro-jejunostomy is performed to reduce gastric acidity and to overcome any pylorospasm.

In Walton's series ${ }^{17}$ of 3 Io patients there were $I_{3}$ deaths -4.2 per cent. In a group, in which a large number of cases were followed up by him for a period of five years or more, good results were claimed in 88 per cent. But wedge-resection can only be safely performed when the ulcer is small or of moderate size, when the stomach can be readily mobilised, and when the lesion is not too near to the cardia; in other words, V-excision is contra-indicated in those cases in which the ulcer is seemingly inaccessible, is large, and is fixed to adjacent structures.

What is thought to be a small gastric ulcer may prove to be a small ulcerating carcinoma, but in practice a high proportion of the small or moderate-sized ulcers will be found to yield to medical treatment in a most gratifying manner.

As the surgeon is called upon to deal mainly with the large callous recalcitrant penetrating lesions, with those that are firmly fused to the pancreas, and with those in which the menace of cancer cannot be excluded, it stands to reason that, as a rule, some type of gastro-duodenal resection will be indicated.

If a small gastric ulcer proves intractable to well-applied medical treatment it then demands radical measures for its cure in view of the possibility of malignancy, and here partial gastrectomy is advised rather than a limited V-excision combined with gastro-jejunostomy.

For small mobile gastric ulcers of the lesser curvature the mortality of partial gastrectomy is as low as or even lower than that of wedge-resection plus gastro-jejunostomy.

\section{(4) Sleeve Resection.}

Sleeve, or segmental, resection has a very limited scope of usefulness, being occasionally employed for hour-glass stomach or bleeding gastric ulcer which involves the mid-gastric region, as it is a simple procedure and is fairly safe in execution. The final results, however, are unquestionably inferior to those which follow the Polya types of gastrectomy, as the motility of the stomach is subsequently interfered with and recurrent constriction or ulceration at the same site is by no means uncommon.

In Claggett's series4 only 50 per cent of the cases investigated by him showed permanently good results.

\section{(5) Partial Gastric Exclusion.}

This is an operation which is sometimes performed for ulcers of the pyloric segment which have deeply penetrated the liver or pancreas and which are associated with a marked degree of 
surrounding inflammation. By this method of Devines, at the first operation the stomach is transected high up in its upper third, and the lower cut end of the stomach is anastomosed to the proximal jejunum, adopting the Hofmeister plan, after which the distal (pyloric) segment is closed. At the second operation some three weeks or so after the exclusion it will be seen that the inflammation about the ulcer has resolved to such an extent that resection of the pyloric cul-de-sac and of the duodenal bulb has become greatly simplified.

\section{(6) Partial Gastrectomy.}

Partial gastrectomy is the operation of choice in cases of chronic gastric ulcer for these reasons:-

(a) It affords the maximum degree of protection against rccurrence of ulceration, haemorrhage, perforation and the subsequent onset of cancer of the stomach.

(b) The immediate and late results are eminently satisfactory and the operation is in every way consistent with sound health and good digestion. The death-rate of partial gastrectomy for gastric ulcer should not exceed 5 per cent, and it is possible, as I have shown, ${ }^{12}$ to operate upon a large series of patients with gastric ulcer with a mortality of less than this. About 90 per cent of the patients show a good all-round efficiency for work and are able to take ordinary diet without discomfort or any untoward symptoms. The unsatisfactory results include a few cases in which microcytic anaemia develops, which, however, can be speedily cured by the administration of ferrous salts and suitable food. Some 2 per cent of the cases develop cancer in the remaining portion of the stomach. The incidence of stomal ulceration is less than 0.5 per cent.

(c) The technique of the operation greatly simplifies the removal of the ulcer. On the other hand, it is well known that V-excision presents many difficulties: the aperture which follows removal of the lesion is not easy to close, and the suturing produces some distortion of the stomach which interferes with its functional capacity, this, however, being in part overcome by the added short-circuiting procedure.

(d) Partial gastrectomy is accepted by the majority of surgeons as being the most successful undertaking in gastric ulcer cases. There is, however, some divergence of opinion as to whether better results follow the Billroth I methods of repair or the Polya types of operation.

On physiological grounds it would seem immaterial whether after an adequate gastric excision the cut end of the stomach were joined to the duodenum or to a loop of proximal jejunum; but on technical grounds and on account of the smoothness of convalescence which attends the Polya methods and of the brilliance of the late results, this latter operation is the one which I have employed with gratifying results for nearly all my cases.

\section{THE TECHNIQUE OF PARTIAL GASTRECTOMY FOR GASTRIC ULCER BY THE POLYA-HOFMEISTER PLAN}

Some four hours or so before the operation is due to commence, a Ryle tube is passed through the nostril into the stomach and the gastric contents are aspirated, after which the stomach is thoroughly irrigated with one pint of 0.8 per cent sulfanilamide in physiological saline. The end of the Ryle tube is strapped to the patient's cheek or forehead, and is left in situ throughout the operation and for the first two or three post-operative days.

The most important person at a gastrectomy operation, after the patient and the surgeon, is, of course, the anaesthetist. Not only must the administration of the anaesthetic be perfect but he must take frequent recordings of the blood pressure, attend to the intravenous drip apparatus to see that it is working smoothly, while in addition he must apply frequent suction to the indwelling tube to keep the stomach completely deflated throughout the course of the operation. It is a most difficult matter to excise a stomach which is tensely blown out with gas or gastric secretion.

There is a choice of three incisions: midline, left paramedian, or left transrectus musclesplitting. For the majority of cases the midline incision, which starts from the tip of the xiphisternum and extends downwards to the umbilicus, usually suffices. Haemostasis must be complete, the blood vessels being tied off with fine silk, after which tetra-cloths are applied to the 
skin margins, and Lahey cellophane squares are affixed to the cut edges of the peritoneum. During this operation it is most important to afford the maximum degree of protection to the vulnerable layers of the abdominal wall, and in practice this method has been found effective.

After conducting a methodical abdominal exploration and having decided that the case is one which is suitable for partial gastrectomy, the operation is commenced by making an opening through a bloodless spot in the gastro-colic omentum, well to the left of the midline. The opening is enlarged and the superior aspect of the mesocolon is stripped away downwards from the posterior wall of the stomach, the adhesions which bind these structures together being gently separated with a swab or with scissors. The middle colic artery and its arching branches should be clearly demonstrated from both aspects of the mesocolon and they should be protected from any harm by placing a large Cripps pad or an abdominal pack into the omental bursa. The gastro-colic omentum is next ligatured piece by piece, tied with No. 6 silk, and divided from the lower border of the spleen to the first part of the duodenum. When the duodenum is reached, the right gastro-epiploic artery is isolated, secured and divided, after which the superior pancreatico-duodenal vessels are underrun, tied off and cut. The first part of the duodenum and pylorus are drawn upwards and outwards to the right, and at the same time rotated so as to display the posterior aspect of the gut, any adhesions here being cautiously ligatured and divided.

The gastro-duodenal artery will now be seen crossing the head of the pancreas beneath its thick covering of peritoneum. The supra-duodenal artery of Wilkie and the right gastric artery are indentified, underrun, ligatured in two places with No. 6 silk and divided. When this has been done, the pylorus and the first part of the duodenum should be quite free and thoroughly mobilised. There is never any difficulty in mobilising the first portion of the duodenum in cases of gastric ulcer unless there is a concomitant posterior-wall duodenal ulcer.

With the first portion of the duodenum mobilised, two enterotomes are applied to the intestine, one inch distal to the pyloric outlet, and the duodenum is divided between the clamps with a cautery or a carbolised knife. The proximal end embraced by the clamp is covered with a mackintosh square and drawn well over to the left side, away from the field of operation. A Deaver retractor is inserted beneath the right margin of the abdominal incision to retract the abdominal wall laterally in order to facilitate the exposure and suturing of the duodenal stump.

There are many ways of closing and turning in the duodenal stump. If there is no tension and ample duodenum to spare, by far the simplest method is by the use of the Mikulicz stitch. After this has been inserted, one or two purse-string sutures of moderately strong silk are introduced and firmly tied. It is safer to use silk for the closure of the duodenal stump rather than catgut, and after the inversion has been completed it is important to protect the suture line with pads of adjacent gastro-hepatic and gastro-colic omenta. I sometimes tack down the peritoneum overlying the head of the pancreas to the anterior aspect of the inverted duodenal stump so as to place this in a retro-peritoneal position. The greater curvature near the lower border of the spleen is now prepared for anastomosis by tying off and dividing the left gastroepiploic artery where it swings round at the lower border of the gastro-splenic omentum. The greater curvature at this point should be quite free of any fatty tabs.

If the ulcer has deeply penetrated the substance of the pancreas, the posterior wall of the stomach around the ulcer should be cut free with an electric cautery, the base of the ulcer being left behind in the pancreatic substance. After disinfecting the surface of the crater it should be methodically destroyed by heat. If the ulcer is attached to the pancreas only by adhesions, these should be isolated and divided between ligatures.

The most difficult part of the operation consists in the high division of the left gastric artery. This is difficult because the gastro-hepatic omentum is here often oedematous, thickened, sclerotic and crowded with swollen lymphatic glands.

A method I frequently employ is to insert the index finger of the left hand high up on the posterior wall of the lesser curvature and with this finger to push forward the blood vessels contained in the gastro-hepatic omentum. An opening is then made between the lesser curvature itself and the vascular bundle by means of the left index finger and thumbs working together. Through this opening a pair of large artery forceps is passed to act as a guide for the strong ligatures which are mounted on aneurysm needles and slipped through this gap. The first ligature is then drawn backwards towards the coeliac axis and tied tightly as close to the aorta 
as possible. The other ligatures are introduced and tied, after which the thick pedicle is cut through between the middle ligature and the one that has been applied close to the lesser curvature of the stomach.

As soon as this pedicle is divided, a raw surface results on the lesser curvature, and this should be reperitonised by introducing a few closely-applied interrupted silk sutures.

A Friedrich-Petz sewing machine is next clamped on to the stomach from the greater to the lesser curvature on the line prepared for the transection, which runs from a point on the lesser curvature about 2 inches below the cardiac orifice and on the greater curvature at a point distal to where the left gastro-epiploic artery has been ligatured. The instrument is forced home and the two rows of fine metal clips are neatly inserted. Between these two rows of clips the stomach is cut across with a cautery and the specimen removed. The upper cut end of the stomach, together with its clips, is inverted with two rows of continuous sutures and one row of interrupted sutures of fine silk, while the lower half of the cut end of the stomach is grasped with Allis forceps and drawn through the wound in readiness for the anastomosis. The duodenojejunal flexure is then visualised and a loop of proximal jejunum some I2-I4 inches long is drawn through the wound, brought over the transverse colon and applied to the gastric pouch from right to left, the most proximal point of the jejunum that is to be used for the anastomosis being applied first to the lesser curvature. The jejunal loop needs to be long, as after the anastomosis is completed the small gastric pouch retracts upwards considerably, and if there is any tension there will be kinking, both at the duodeno-jejunal flexure and at the proximal point of the anastomotic line.

The jejunum should curve gracefully from the duodeno-jejunal flexure to the reconstructed lesser curvature of the stomach, and there must be a fair degree of slack. The anastomosis is now made, using four rows of sutures anteriorly and posteriorly, the first and the last being of interrupted silk, the rest being continuous sutures of No. 00 20-day chromic catgut.

When the anastomosis is completed and haemostasis is assured, the jejunum just proximal to the point of anastomosis is buttressed against the inverted and newly-reconstructed lesser curvature of the stomach, not only to protect this vulnerable portion of the suture line but also to reinforce the Hofmeister valve which has been fashioned. This valve and the obliquity with which the stoma has been constructed ensure that all nourishment is directed downwards into the distal jejunum and that none finds its way into the duodenal cul-de-sac. Such unpleasant symptoms as bloating, colicky epigastric pain, a sensation of tightness after meals, and regurgitation of bile into the mouth are due to food being dumped into the duodenum.

The operation is completed by reinforcing the anastomotic line at the greater curvature with an adjacent portion of the gastro-colic omentum and by stitching remnants of gastrohepatic omentum to the superior angle of the stoma. These fatty reinforcements also act as slings and help to relieve any strain on the line of anastomosis.

The abdominal wound is closed with a continuous suture which picks up all the layers of the abdominal wall except the skin and subcutaneous tissues, using fine chromic catgut reinforced with a few interrupted sutures of floss silk. The skin margins themselves are approximated with Michel clips or with deknatel or silk sutures. The wound is then painted with iodine and covered with a thin layer of gauze which is held in place with strips of Elastoplast.

It will be noted that no enterostomy clamps are employed in fashioning the anastomosis. It is, in my opinion, quite impossible to perform a really high gastrectomy if enterostomy clamps are used.

\section{SUMMARY}

(I) All patients suffering from chronic gastric ulcer should be admitted to hospital for investigation and a course of medical treatment.

(2) A scheme of management for these cases is briefly outlined.

(3) The indications for operation are:-

(a) The presence of complications;

(b) The failure of prolonged and efficient medical treatment; and

(c) Inability to exclude the possibility of malignancy. 
(4) The relative merits of the various operations which have been employed in the treatment of gastric ulcer are discussed.

(5) The operation of choice is partial gastrectomy, preferably by the ante-colic HofmeisterPolya plan. This operation is associated with a mortality which does not exceed 5 per cent and the late results are eminently satisfactory in over 90 per cent of the cases thus treated. The ante-colic method is preferred to the retro-colic or posterior Polya procedure as it is simpler to perform, the immediate and late results are better, and should anything perchance go wrong with the anastomosis, such as the late development of a gastro-jejunal ulcer, it is far easier to tackle the lesion. given.

(6) A short account of the operation of partial gastrectomy as practised by the author is

\section{REFERENCES}

1. BALFOUR: Surg. Clin. N. Amer. 1. 5, 1921.

2. Ann. Surg. 76. 405. 1922.

3. CHAM̈BERLAIN: Surg., Gyne., and Obst. 451. 183.1927.

4. CLAGGETT: Proc. Staff Meet. Mayo Clin. 15. 337. 1940.

5. DEVINE: Surg., Gynec. and Obst. 40. 1. 1925.

6. EINHORN: Med. Rec. 96. 95. 1919.

7. EUSTERMAN and BALFOUR: The Stomach and Duodenum. Saunders Co., London. 1935.

8. FINSTERER: Proc. Roy. Soc. Med. 32. 183.1939.

9. HURST: Post-Graduate Surgery. (Ed. Rodney Maingot) 1. 576. 1936. Med. Publs. Ltd., London.

10. KATSCH: (Cited by Hurst). Lancet. 2. 1455. 1939.

11. LAHEY: New Eng. J. Med. 220. 315. 1939.

12. MAINGOT: Abdominal Operations. 1. 290. 1940. Appleton-Century, London.

13. STEWART: HURST \& STEWART. Gastric and Duodenal Ulcer. Oxford Med. Publs., London. 1929.

14. SPRIGGS: Brit. Med. J. 2. 39.1940.

15. WALTMAN WALTERS \& CLEVELAND: Minnesota Med. 23.709 .1940$.

16. WALTMAN WALTERS: Arch. Surg. 41. 542.1940.

17. WALTON: Prognosis. 1. 109. 1935. Lancet Ltd., London.

\section{New and Recent Publications from Heinemann}

GEÖRGE BANKOFF, M.D., D.Ch., F.R.F.P.S.

The Practice of Local Anaesthesia

A concise and practical manual covering technique for all parts of the body, and specially intended for surgeons without special skill or knowledge of the subject.

200 pages. 121 photographs and diagrams. $17 s .6 d$.

\section{LEOPOLD LICHTWITZ, M.D. Functional Pathology}

After thirty years of clinical and laboratory experience, the author, now professor of medicine at Columbia University, gives a comprehensive survey of the mechanism of internal diseases, dealing with signs, symptoms and treatment. "The book has excellent photographs, useful tables and charts, and should fulfl the author's wish to stimulate discussion." -The Lancet.

567 pages.

157 illustrations.

42s.

K. C. CLARK, F.S.R.

Positioning in Radiography THIRD EDITION

After just over three years, this incomparable atlas of radiographic technique has again been revised and slightly enlarged for a third edition.

Now 70s.

\section{GRANTLY DICK READ, M.A., M.D. Revelation of Childbirth}

Well known for his pioneer work on a new attitude to the management of pregnancy and labour, Dr. Dick Read here provides a full-length work which is both a record of experience and a clear statement of aims and methods. Physicians, midwives and nurses will find this new work invaluable as a supplement to the standard textbooks of obstetrics. 270 pages.

\section{A. PINEY, M.D., M.R.C.P. Synopsis of Blood Diseases}

"Altogether we consider this to be far and away the best guide to the study of blood diseases ever published. Although it is called a synopsis, it is much more, and anyone who masters its contents will possess a really practical knowledge of diseases of the blood."-The Prescriber.

120 pages. 4 plates and glossary.

10s. 6d.

J. G. BONNIN, M.B., B.S., F.R.C.S.

A Complete Outline of Fractures

"A really practical and useful volume which should strongly appeal to students, practitioners and to medical men in the Services."-Journal of the Royal Naval Medical Service.

507 pages $\quad 575$ illustrations.

27s. $6 d$.

WILLIAM HEINEMANN • MEDICAL BOOKS• LTD., 99 GREAT RUSSELL ST., LONDON, W.C.1 